\section{ORIGINAL RESEARCH}

W.E. Reddick

J.O. Glass

D.P. Johnson

F.H. Laningham

C.-H. Pui

\title{
Voxel-Based Analysis of T2 Hyperintensities in White Matter during Treatment of Childhood Leukemia
}

BACKGROUND AND PURPOSE: White matter (WM) hyperintensities on T2-weighted MR imaging are the most common imaging manifestation of neurotoxic effects of therapy for central nervous system (CNS) prophylaxis in childhood acute lymphoblastic leukemia (ALL). This study uses voxel-based analyses (VBA) of T2-weighted imaging of patients during treatment to identify which WM regions are preferentially damaged.

MATERIALS AND METHODS: Two sets of conventional T2-weighted axial images were acquired on a 1.5T MR imaging scanner from 197 consecutive patients (85 female, 112 male; aged 1.0-18.9 years) enrolled on an institutional ALL treatment protocol. Images were acquired after completion of induction therapy and after the final of the 4 courses of intravenous high-dose methotrexate in consolidation therapy ( $3.9 \pm 0.8$ months apart). Voxel-wise statistical testing of the incremental change between normalized longitudinal T2 images was performed with radiologist reading (normal or abnormal) and treatment risk-group as covariates.

RESULTS: Two highly significant bilateral clusters of T2 signal intensity change were identified in both 1-group and 2-group analyses. The regions were symmetric in size, shape, and average signal intensity. Increased T2-weighted signal intensity from these regions both within and between examinations were nonlinear functions of age at examination, and the difference between the examinations was greater for older subjects who received more intense therapy.

CoNCLUSIONS: These analyses identified specific WM tracts involving predominantly the anterior, superior, and posterior corona radiata and superior longitudinal fasciculus, which were at increased risk for the development of T2-weighted hyperintensities during therapy for childhood ALL. These vulnerable regions may be the cause of subsequent cognitive difficulties consistently observed in survivors.

A cute lymphoblastic leukemia (ALL) is the most common childhood cancer, accounting for 1 of 4 childhood cancers. With contemporary effective treatment, the 5-year event-free survival estimate for pediatric patients with ALL is approximately $80 \% .{ }^{1}$ Methotrexate given intravenously (IVMTX) at high dose has been shown to decrease the incidence of hematologic, testicular, and central nervous system (CNS) relapse. However, methotrexate can have significant adverse toxic effects on the CNS, resulting in severe neurologic morbidity. Leukoencephalopathy (LE), seen as white matter (WM) hyperintensities on T2-weighted MR imaging, is the most common imaging manifestation and may be either persistent or transient. ${ }^{2}$

A recent study of childhood ALL survivors objectively assessed the relationship between MR measures of WM volume and neurocognitive performance. ${ }^{3}$ Survivors demonstrated significantly less WM volume than age-matched control sub-

Received March 31, 2009; accepted after revision May 14.

From the Divisions of Translational Imaging Research (W.E.R., J.O.G., D.P.J.) and Diagnostic Imaging (F.H.L.) within the Department of Radiological Sciences, and Department of Oncology (C.-H.P.), St. Jude Children's Research Hospital, Memphis, Tenn.

This work was supported in part by R01-CA90246 and by Cancer Center Support grant P30-CA21765 from the National Cancer Institute, by the American Cancer Society FM Kirby Clinical Research Professorship, and by the American Lebanese Syrian Associated Charities.

Please address correspondence to Wilburn E. Reddick, PhD, Division of Translational Imaging Research (MS \#220), Department of Radiological Sciences, St. Jude Children's Research Hospital, 262 Danny Thomas Pl, Memphis, TN 38105-3678; e-mail: gene.reddick@stjude.org

Indicates open access to non-subscribers at www.ajnr.org

DOI 10.3174/ajnr.A1733 jects indicating atypical WM maturation or damage. A consistent pattern of attention changes in both the specific attention measures and the freedom from distractibility index in the intelligence scales was demonstrated and was associated with lower WM volumes.

Other studies have established the prevalence, extent, and intensity of LE in patients during treatment of ALL by using objective quantitative MR imaging measures. ${ }^{4,5}$ These studies demonstrated that increasing intensity of therapy, corresponding to more courses and higher doses of IV-MTX, was associated with an increased prevalence, intensity, and extent of LE. Most of the LE was transient, but the impact of these changes on the developing brain are unknown. ${ }^{6}$

A better understanding of the specific WM tracts at increased risk for injury during therapy will elucidate more specific neurocognitive domains supported by these tracts. This knowledge would form the basis to identify specific subsets of patients early in therapy who may be at increased risk for injury and may benefit from interventions focused on specific neurocognitive deficits. However, an objective assessment of the spatial localization of WM affected by LE during treatment of ALL has yet to be performed. This study uses voxel-based analyses (VBA) of T2weighted imaging within the WM of patients after the first and final courses of IV-MTX treatment of ALL to identify which WM tracts were preferentially damaged.

\section{Materials and Methods}

\section{Patient Population}

Consecutive patients aged at least 1 year enrolled on an institutional ALL treatment protocol from June 2000 through September 2005 
were eligible for the study. ALL treatment was based on a comprehensive risk classification, including blast cell immunophenotype and genotype, presenting clinical features, and early treatment response. Patients were assigned to 1 of 3 risk groups: low-risk (LR) and standard-risk or high-risk (SHR). Patients between 1 and 10 years old with B-cell precursor and presenting leukocyte count $<50 \times 10^{9} / \mathrm{L}$, leukemic cell DNA index of 1.16 or higher, or TEL-AML1 fusion were provisionally classified to have low-risk ALL, provided that they did not have testicular or CNS leukemia (ie, CNS3 status), hypodiploidy ( $<45$ chromosomes), E2A-PBX1 fusion, or MLL rearrangement. ${ }^{7}$ Patients with $B C R-A B L$ fusion (Philadelphia chromosome) were designated to have high-risk disease, and all others including patients with T-cell ALL were provisionally classified to have standard-risk ALL. Final risk status depended on the response to remission-induction therapy. Any patients with $0.01 \%$ to $0.99 \%$ residual leukemia after completion of 6-week induction therapy were considered to have standard-risk ALL and received intensive postremission therapy, whereas those with $1 \%$ or more residual disease were designated to have high-risk ALL and were candidates for allogeneic hematopoietic stem cell transplantation.

During consolidation therapy, LR patients received IV-MTX at $2.5 \mathrm{~g} / \mathrm{m}^{2}$ plus intrathecal therapy every other week for 4 doses, whereas SHR patients received $5.0 \mathrm{~g} / \mathrm{m}^{2}$ together with intrathecal therapy. No patients received craniospinal irradiation. Written informed consent was obtained from the patient, parent, or guardian according to the Institutional Review Board, National Cancer Institute, and Office for Human Research Protections guidelines.

A single radiologist (F.H.L.) retrospectively reviewed imaging from the 287 subjects enrolled on the treatment protocol during the specified period who had completed therapy. The radiologist viewed all longitudinal imaging available from each patient throughout therapy and took into account the patients' age at the time of examination. He used imaging from young healthy control subjects to assist in differentiating typical age-appropriate $\mathrm{T} 2$ hyperintensities in the terminal zones from those changes that were more likely therapy induced. Therapy-induced changes were assessed according to the Common Toxicity Criteria v2.0 for leukoencephalopathy, which requires focal or diffuse T2 hyperintensities involving the periventricular, centrum semiovale, or other susceptible WM areas of the cerebrum and an increase in subarachnoid space possibly with mild ventriculomegaly.

To be eligible for this study, patients had to complete MR imaging examinations at baseline after induction therapy and after the final course of IV-MTX in consolidation therapy. The differences in imaging between these 2 time points represent changes that occur during consolidation therapy. Eighty-four patients were missing at least 1 of the 2 examinations. Six additional patients with imaging findings of cerebral thrombosis, encephalomalacia, or large developmental abnormalities were also excluded from the study. These exclusion criteria resulted in a final cohort of 197 subjects ( 85 female; 112 male: 108 LR and 89 SHR aged 1.0-18.9 years [median, 5.3 years] at diagnosis. Time between the 2 examinations was $3.9 \pm 0.8$ months.

\section{MR Imaging}

Imaging was performed on 1 of $21.5 \mathrm{~T}$ whole-body MR systems with use of the standard circular polarized volume head coil (Avanto; Siemens, Iselin, NJ). No hardware upgrades were performed during the conduct of this study. Software upgrades did occur during the study, but their impact on the routine T2-weighted imaging sequence used was negligible. Conventional imaging was acquired and included at least 19 4-mm-thick axial T1-weighted, T2-weighted, proton density (PD)-weighted, and fluid-attenuated inversion recovery (FLAIR)weighted imaging sets, which covered most of the cerebrum starting at the apex of the brain but did not include the cerebellum because of limitations of coverage in the FLAIR sequence. The T2 imaging set has higher signal-to-noise intensity and less artifacts than the FLAIR imaging and was selected for this study of T2-hypertintensities within the WM. Imaging was acquired as a T2-weighted, PD-weighted dual spin-echo image set (TR, $3500 \mathrm{~ms}$; TE1, $17 \mathrm{~ms}$; TE2, $102 \mathrm{~ms} ; 7$ echoes). Unfortunately, the imaging acquired in this treatment protocol, which began in 2000, did not include diffusion tensor imaging as a standard part of clinical imaging.

\section{Spatial Normalization}

VBA require that all examinations be registered into a common stereotactic space. For this study, each patient examination was registered to the ICBM T2 50 atlas (http://www.loni.ucla.edu/Atlases). This atlas is an average of the T2-weighted MR images of 50 healthy young adult brains in the deterministic atlas space of the ICBM 452 and not on any single subject. It represents the mean volume constructed from the average position, orientation, scale, and shear from all 50 individual subjects.

We achieved the spatial normalization of patient examinations to the T2 atlas using a free-form deformation (FFD) with B-spline interpolation, providing local support to model large focal warping ${ }^{8}$ included in the VTK CISG Registration Toolkit 2.0. ${ }^{9}$ To more closely match the $\mathrm{T} 2$ atlas, extrameningeal tissues were removed before registration with use of custom software on the basis of image intensity gradients. Default values for the FFD were used in all normalizations. The deformation field was determined by the displacements specified on a lattice of control points, and the displacements that were off the control points were interpolated on the basis of the neighborhood control points with use of uniform cubic B-splines. Normalized mutual information was used to measure image similarity during normalization with the FFD.

\section{$V B A$}

We performed voxel-wise statistical testing of the normalized longitudinal T2 images using the VBM Toolkit implemented within SPM2 (Wellcome Department of Imaging Neuroscience, London, UK; http://www.fil.ion.ucl.ac.uk/spm/). VBA is a widely used statistical image analysis approach for the detection of WM abnormalities that spatially normalizes (co-registers) brain images across subjects and performs statistical tests at each voxel. ${ }^{10-12}$ The advantages of VBA are that it is highly reproducible, user independent, and it can explore differences over the entire brain without anatomically specific hypotheses. In preparation for this analysis, a WM mask was created from the average of normalized T2-weighted images from the first examination with use of SPM2 to segment WM and then threshold the resulting image at a level of 0.7 probability to generate a binary WM mask. Second, the normalized T2 examinations were all smoothed with a full width half maximum 5-mm Gaussian kernel.

Longitudinal voxel-wise analyses were performed for 1 group with 2 conditions (examinations): after induction therapy and after the final course of IV-MTX. The radiologist reading of the examinations as normal (0) or abnormal (1) was included as a covariate. No nuisance variables were included, and a default 0.1 threshold was used in the analysis, along with the explicit mask of WM. Analyses were performed for all patients as a single group and then as a 2-group analysis stratified by treatment risk-group (LR and SHR). Age at the time of 


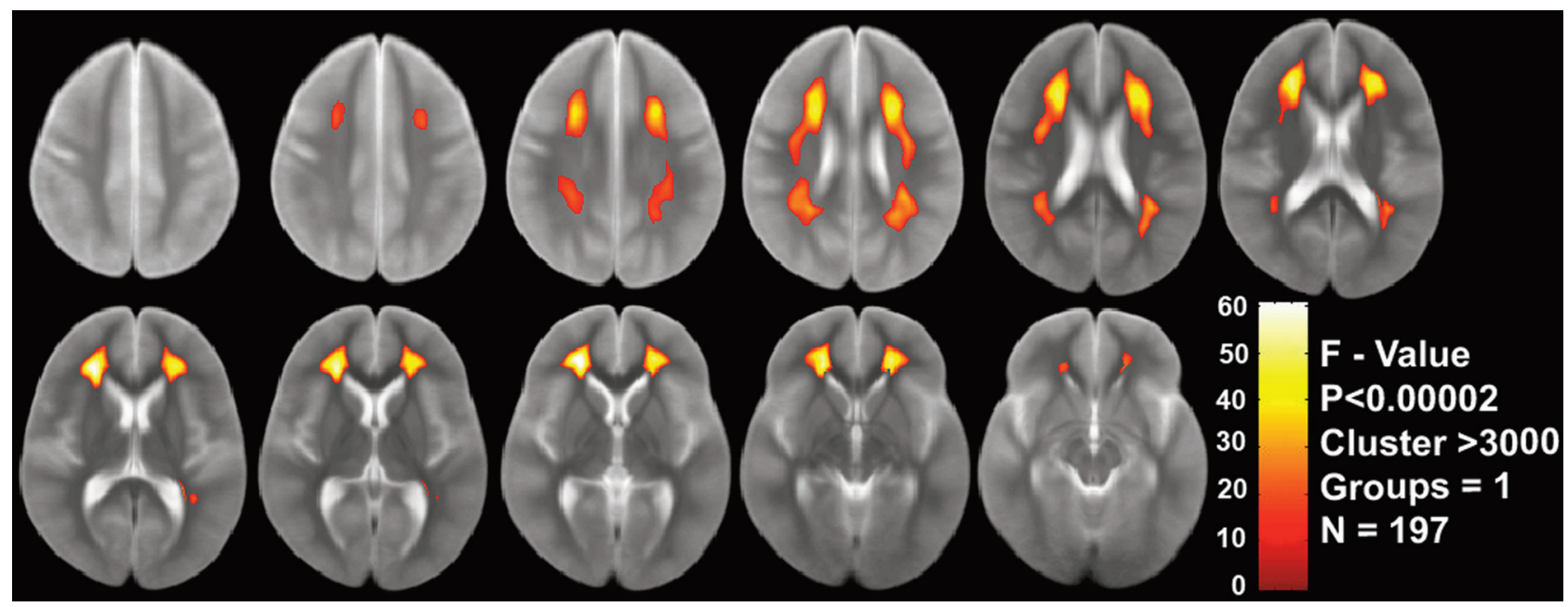

Fig 1. VBA results from the 1-group analysis of all patients $(n=197)$ regardless of treatment risk group are displayed on the average of the normalized T2-weighted images from the first examination for visualization of the spatial localization and extent of the regions.

examination was not included in the analysis because it correlates highly with treatment risk-group.

\section{Statistical Analyses}

$F$-tests were performed to determine which voxels were significantly different between examinations over the subject populations. A $P$ value and cluster threshold were specified to limit the analysis only to regions that have significant differences between examinations and have a sufficient number of continuous voxels for analysis. A false discovery rate approach, which controls the expected proportion of false-positives among suprathreshold voxels, was used to account for multiple comparisons. ${ }^{13}$ The results were overlaid onto the average of normalized T2-weighted images from the first examination for visualization.

\section{Results}

\section{One-Group Analysis}

The first analysis performed was for all 197 patients in the study regardless of risk stratification. Almost one third of the second examinations (57 [29\%] of 197) were read by the radiologist as exhibiting T2 hyperintensities. It should be noted that 3 additional patients who tested normal on the early examinations went on to have T2 hyperintensities later in therapy. The large size of this sample enabled a quantification of spatial regions within the brain, which were significantly different on the second examination compared with the first examination of the same patients. There were 4 potential patterns for radiologist readings of these 2 examinations: normal on both, abnormal on both, normal then abnormal, or abnormal becoming normal. Given the timing of the MR examinations relative to therapy, it was not anticipated that the results of any patient examination would be abnormal after induction and would become normal after the more intensive consolidation phase of therapy. Indeed, none of the 197 subjects exhibited this pattern. As would also be expected, most of the patients' results were read as normal on both examinations (140 [71\%] of 197). Of the remaining patients, 31 (16\%) of 197 exhibited T2 hyperintensities on the second examination only and 26 (13\%) of 197 exhibited changes on both examinations.

Figure 1 demonstrates the voxels that were significantly different at a level of $P<.00002$ with a cluster threshold of 3000 voxels. The $F$-values displayed in the colored clusters have a maximal value of 60 . Two large contiguous clusters of approximately the same size and spatial extent were identified ( $n=19,329$ voxels on the left and $n=18,689$ voxels on the right). These highly significant clusters were most prominent in the frontal lobes and extended superiorly over the ventricles into the posterior parietal lobes. These regions seemed to involve predominantly the anterior, superior, and posterior corona radiata and superior longitudinal fasciculus fiber tracts.

\section{Two-Group Analysis}

Because the changes during consolidation therapy demonstrated in the first analysis could vary with intensity of therapy and potentially age at treatment, a second 2-group analysis, stratified by treatment risk-group, was performed. Age at the time of treatment was not included in the analysis because it correlated highly with treatment risk-group $(R=0.405 ; P<$ $.001)$. The LR patients were an average age of $5.1 \pm 3.1$ years at diagnosis, whereas the SHR patients had an average age of $8.6 \pm 4.8$ years at diagnosis. There was no substantial difference in the proportion of examinations read by the radiologist as exhibiting T2 hyperintensities between the 2 groups, with $28 \%$ of LR subjects and $30 \%$ of SHR subjects read as abnormal.

Figure 2 demonstrates the voxels that were significantly different at a level of $P<.0001$ with a cluster threshold of 1000 voxels. The $F$-values displayed in the colored clusters have a maximal value of 8 , which is substantially lower than the 1-group analysis as expected. Three large clusters were identified, with 2 on the left corresponding to approximately the same size and spatial extent as the 1 on the right $(n=16,302$ voxels on the anterior left, $n=3893$ voxels on the posterior left, and $n=25,023$ voxels on the right). As with the 1-group analysis, these significant clusters were most prominent in the frontal lobes and extended superiorly over the ventricles into the posterior parietal lobes involving predominantly the corona radiata and superior longitudinal fasciculus fiber tracts. 


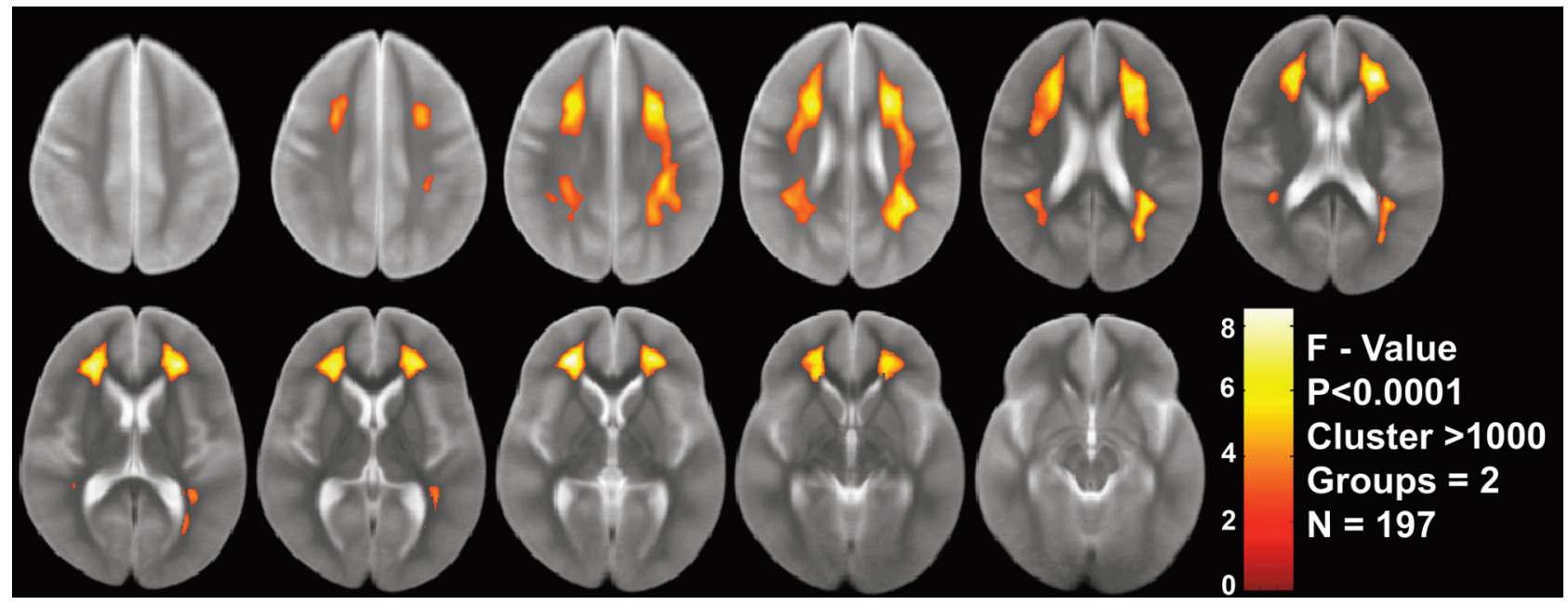

Fig 2. VBA results from the 2-group analysis (LR vs SHR) of all patients ( $n=197)$ are displayed on the average of the normalized T2-weighted images from the first examination for visualization of the spatial localization and extent of the regions.

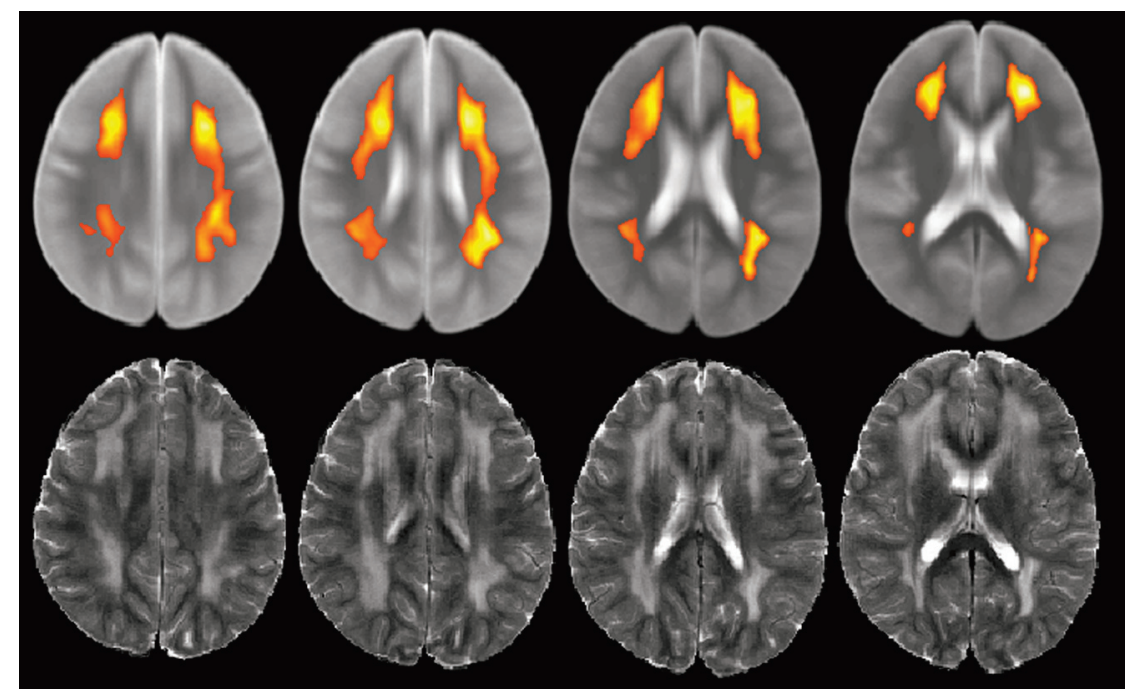

Fig 3. Patient examination after completion of IV-MTX demonstrating T2-hyperintensities within the corona radiata. The regions identified by the VBA are shown for visual comparison.

\section{Representative Patient Examination}

A representative patient examination is shown in Fig 3. This patient was 3.4 years of age at diagnosis and was treated on the LR group of the protocol. Results of the first MR imaging examination were normal, and the second examination exhibited extensive T2 hyperintensities. Representative sections from the normalized T2-weighted second examination are shown in the figure, along with the corresponding clusters identified by the 2-group VBA at the same spatial locations. It can be readily appreciated that almost all of the T2 hyperintensities seen in this patient are within the locations identified by the VBA. Some areas of hyperintensities for this individual patient examination are larger than the clusters from the VBA. The result of the VBA is a map that identifies the most significant regions exhibiting T2 hyperintensity changes during consolidation therapy and is based on population differences that will not map exactly to every individual patient. However, individual examinations with T2 hyperintensities should exhibit a high degree of spatial overlap with the regions identified by the VBA.

\section{Characteristics of Individual T2 Signal Intensity within the VBA Clusters}

A series of analyses were conducted to characterize the individual changes in $\mathrm{T} 2$ signal intensity within the VBA identified clusters for symmetry and changes within and between examinations as functions of age at examination. The 2 clusters identified in the 1-group analysis were similar in size and shape, with less than $3 \%$ difference in size. Analysis of the average signal intensity from each of these clusters also proved to be highly symmetric as shown in Fig 4. A linear regression analysis of each examination with a zero intercept demonstrated unity slopes with correlation coefficients greater than 0.98 . On the basis of this high degree of symmetry in signal intensity, we combined data from the 2 clusters for additional analysis as a function of age at examination.

Figure 5 demonstrates the nonlinear distribution of average T2 signal intensity from the VBA clusters for both examinations on all 197 subjects plotted against age at examination. Data from each examination were then assessed with a logistic regression to demonstrate the longitudinal change in T2 signal 

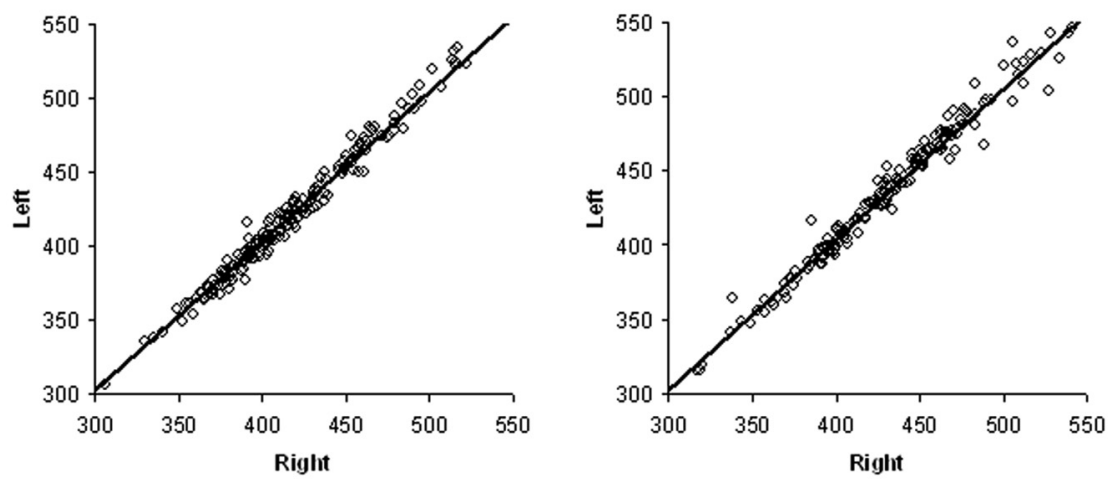

Fig 4. Average T2 signal intensity from VBA clusters on first (left) and second (right) examinations for all 197 subjects to assess symmetry between clusters from each hemisphere. Linear regressions demonstrate slopes of unity with correlations of 0.982 and 0.984 , respectively.

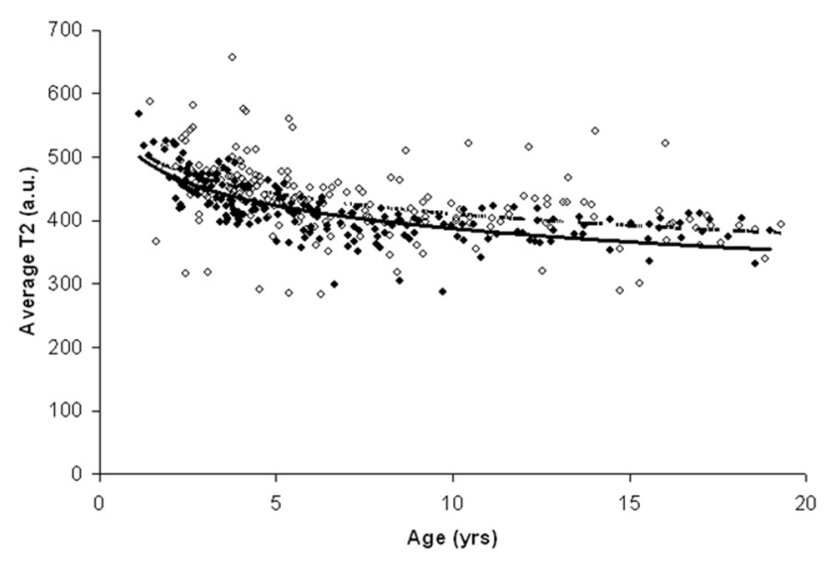

Fig 5. Average T2 signal intensity from VBA clusters on first (filled circle) and second (open circle) examinations for all 197 subjects as a function of age at examination. Signal intensities from both left and right cluster were combined. Logistic regressions are shown for both examinations (solid, first examination; dashed, second examination).

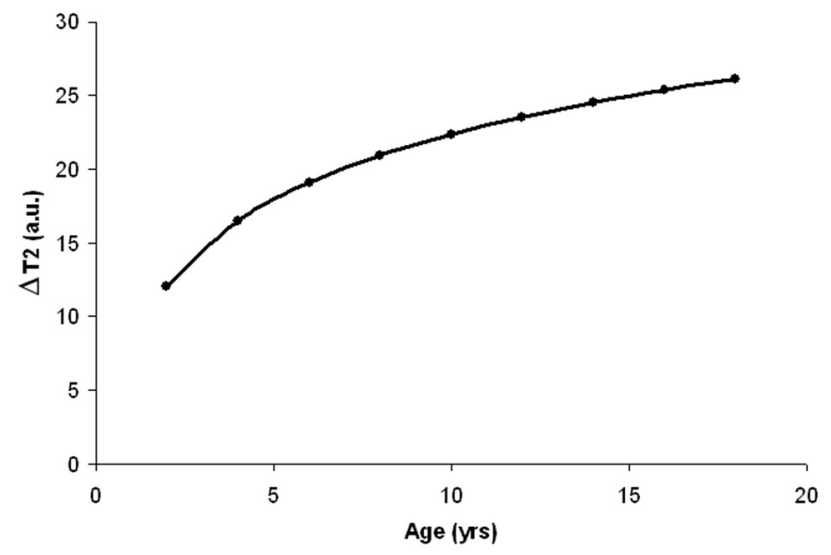

Fig 6. Difference in T2 signal intensity between the 2 examinations on the basis of the logistic regressions determined in Fig 5. The logistic regressions from each examination were evaluated for ages ranging from 2 to 18 years, and the differences between values at each age are demonstrated.

intensity. The 2 regression curves diverge with increasing age at examination so that the increase in signal intensity on the second examination appears greater for older subjects. To more clearly illustrate this affect, Fig 6 was generated on the basis of differences in signal intensity calculated from the 2 logistic regressions evaluated at ages ranging from 2 to 18 years. It was evident from the plot that the change in T2 signal intensity between examinations was twice as great for the 18year-old compared with the 2-year-old. Furthermore, this effect of age at examination on T2 signal intensity change during therapy was nonlinear.

\section{Discussion}

This study identified 2 highly significant bilateral clusters of T2 signal intensity change in WM among patients treated for ALL. The regions were symmetric in size, shape, and average signal intensity. These clusters were most prominent in the frontal lobes and extended superiorly over the ventricles into the posterior parietal lobes involving predominantly the anterior, superior, and posterior corona radiata and superior longitudinal fasciculus fiber tracts. Changes in average signal intensity from these regions both within and between examinations were nonlinear functions of age at examination. T2 signal intensity was greater on the second examination, and the difference between the first and second examination was greater for older subjects. Similar regions were also identified on a 2-group analysis, which controlled for treatment risk-group.

Neuroimaging studies have consistently reported morphologic changes in the cerebrum of children treated for ALL without radiation therapy. ${ }^{3,14-18}$ Most of these studies have focused on WM changes, particularly in the frontal lobes. ${ }^{3,18}$ Most recently, a whole brain voxel-based morphometry study of ALL survivors treated without irradiation was compared with healthy control subjects. ${ }^{19}$ The study found 2 distinct clusters of reduced WM volume in the right frontal lobe. The region identified in the right middle frontal gyrus of these long-term survivors is encompassed within the spatial regions identified in the current VBA of T2-hyperintensity during treatment.

A recent review by Reddick et $\mathrm{al}^{6}$ included a VBA analysis of patients during therapy for ALL stratified by age at diagnosis but did not control for treatment group (intensity of therapy), which correlated highly with age at diagnosis. In the current longitudinal study, all 197 subjects are used in the VBA, and treatment group is included as a covariate, thus increasing the power of the study and resulting in increased statistical significance as demonstrated by the $P$ values achieved. This improved approach also has the advantage of resulting in a single outcome (region of interest) across all subjects rather than 2 results depending on the age of the patient. Another advantage 


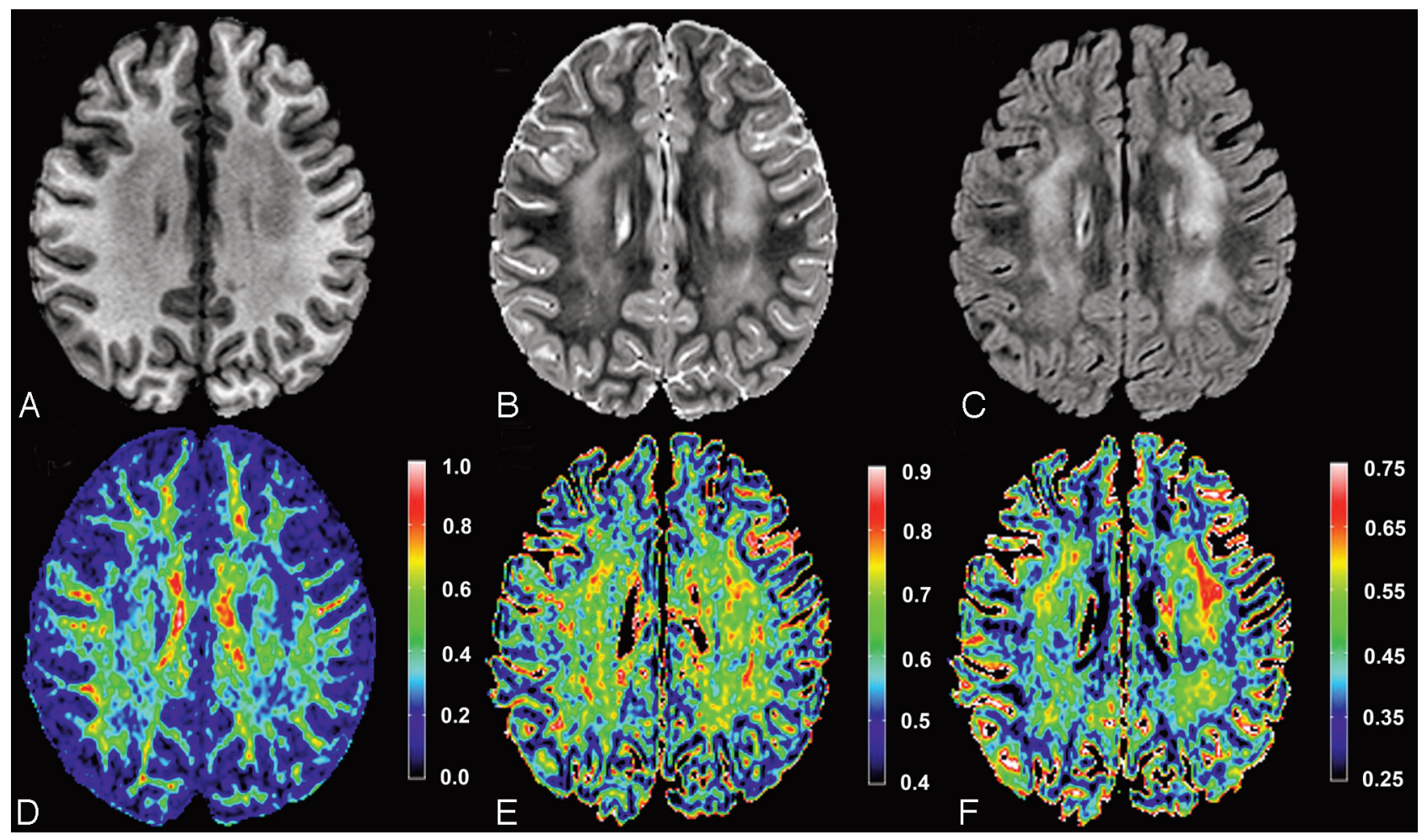

Fig 7. Imaging from a 7-year-old female patient treated for ALL. T1-weighted, T2-weighted, and FLAIR images ( $A-C$, respectively) are shown at an axial level, which demonstrated the hyperintensities within the corona radiata. Corresponding $F A$, axial, and radial diffusivity maps ( $D-F$, respectively) are shown at the same level. Color bars are shown beside each diffusion tensor imaging parameter map for reference. FA values are unitless, whereas axial and radial values are in micrometers squared per second.

of the current study is the use of the high-resolution ICBM T2 50 atlas (1-mm isotropic) as opposed to a single-subject examination with lower-resolution 4 -mm-thick $2 \mathrm{D}$ imaging. The higher resolution target facilitated improved spatial normalization of the patient examinations, resulting in improved sensitivity to longitudinal WM changes. The deterministic atlas space also facilitated the identification of the corona radiata and superior longitudinal fasciculus as fiber tracts involved in the WM abnormality.

Changes in the frontal WM tracts identified by the current VBA could be the cause of subsequent cognitive difficulties in this cohort. Additional changes in the posterior WM tracts may indicate a difference in susceptibility of WM with varying degrees of myelination. Maturation, growth, and organization of regional WM, specifically that in the frontal-parietal regions, play an important role in cognitive development. ${ }^{20}$ ALL survivors exhibit a consistent pattern of deficits in attention/ executive functions, memory (particularly nonverbal), and processing speed, ${ }^{21-25}$ which are often associated with frontal lobe domains ${ }^{17,26}$ and are specifically supported by the fiber tracts implicated in this study.

While evaluating the potential impact of these WM changes, one must consider the potentially confounding effect of age at examination on the interpretation of the results. Figures 5 and 6 demonstrate an increasing difference in T2 signal intensity between the examinations for older subjects. Because older ages are associated with high-risk leukemia and, hence, more intensive therapy such as higher doses of methotrexate, we cannot definitively determine if the increasing difference is because of age or intensity of therapy. Although older age cannot be excluded as a potential confounding variable, most studies of neurotoxicity have determined that younger age at therapy is more often associated with increased risk for damage. Furthermore, increased intensity of CNS-directed therapy is often associated with more imaging and neurocognitive difficulties in survivors.

Another potential limitation of this study was the use of the ICBM T2 atlas, which is based on imaging of young adults. Our patient cohort had a median age of only 5.3 years and included children as young as 1 year. The T2 signal intensity of WM across the full age range included in this study changed by almost $25 \%$. A potential of error is associated with the spatial normalization of young undeveloped brains to an adult atlas. The accuracy of the FFD spatial normalization for pediatric cancer patients was established by Zhang et $\mathrm{al}^{9}$ as an average 2-mm error over all predefined manual landmarks. The magnitude of this error is well below the full width half maximum 5-mm Gaussian kernel used to smooth the examinations. To assess the performance of the spatial normalization, each examination was verified visually for gross distortions or misalignments. Although this does not eliminate the possibility of more subtle misalignments of cortical structures, the alignments of deep WM regions were less likely to be affected. This assessment is consistent with the clarity of the average fractional anisotropy (FA) image produced across all subjects.

Methotrexate has been the primary focus of many studies of neurotoxicity and adverse neurocognitive outcomes. ${ }^{27-29}$ The dose, cumulative exposure, and infusion rate of methotrexate have each been associated with the degree of neurocognitive deficit. ${ }^{27,30}$ Methotrexate, however, is not the only drug used in the treatment of ALL that can adversely affect the mor- 
phologic features of the developing brain. ${ }^{31}$ For example, asparaginase may result in cerebrovascular disease, especially venous sinus thrombosis. Corticosteroids such as dexamethasone may also have a pronounced effect on cerebral morphologic features.

Although this prospective study focused on the conventional T2-weighted MR signal intensity changes, another more specific approach to characterize microstructural changes in WM during therapy is diffusion tensor imaging, which quantifies the diffusion of water in tissue. ${ }^{32}$ Clinical studies of childhood ALL and brain tumors have demonstrated that WM FA was reduced following treatment, and that it is more sensitive than conventional T2-weighted MR imaging in the detection of WM injury. ${ }^{33,34}$ The use of diffusion tensor imaging in asymptomatic patients during or after treatment of ALL is a more recent development. The association of WM FA and IQ in 18 ALL survivors and age-matched healthy control subjects has been prospectively tested. ${ }^{34}$ The FA in the WM of ALL survivors was often lower than that in the age-matched control subjects, and the differential between the FA in patients relative to that in control subjects was directly proportional to the full-scale, performance, and verbal IQ scores of the survivors. An ongoing longitudinal study of diffusion tensor imaging as a clinically useful approach for the assessment of treatment-related neurotoxicity is being investigated in regions identified by the current VBA analysis. An example of conventional and diffusion tensor imaging from a young patient during therapy for ALL is demonstrated in Fig 7. This imaging is from a time point in therapy that is approximately the same as the second time point in the current study. The region of hyperintensity is again noted in the corona radiata and displays decreased FA, only slight elevation of axial diffusivity, and markedly increased radial diffusivity. This pattern is consistent with a transient demyelination and needs to be investigated prospectively.

\section{Conclusions}

In conclusion, this study identified 2 highly significant bilateral clusters of T2 signal intensity change in WM among patients during treatment of ALL with use of both 1-group and 2-group analyses controlling for treatment risk-group. The regions were symmetric in size, shape, and average signal intensity involving predominantly the anterior, superior, and posterior corona radiata and superior longitudinal fasciculus fiber tracts. Changes in average T2-weighted signal intensity from these regions both within and between examinations were nonlinear functions of age at examination, and the difference between the examinations was greater for older subjects who received more intense therapy. Whole-brain VBA identified specific WM tracts that seem to be at increased risk for the development of T2-weighted hyperintensities during therapy for childhood ALL and may be the cause of subsequent cognitive difficulties in survivors. Additional studies will be needed to more fully test this relationship and should include diffusion tensor imaging.

\section{Acknowledgment}

We thank Chiquila Jones for her efforts in processing and analysis of the MR imaging examinations.

\section{References}

1. Pui C-H, Evans WE. Treatment of acute lymphoblastic leukemia. N Engl J Med 2006;354:166-78

2. Shuper A, Stark B, Kornreich L, et al. Methotrexate-related neurotoxicity in the treatment of childhood acute lymphoblastic leukemia. Isr Med Assoc J 2002;4:1050-53

3. Reddick WE, Shan ZY, Glass JO, et al. Smaller white matter volumes are associated with larger deficits in attention and learning among long-term survivors of acute lymphoblastic leukemia. Cancer 2006;106:941-49

4. Reddick WE, Glass JO, Helton KJ, et al. Prevalence of leukoencephalopathy in children treated for acute lymphoblastic leukemia with high-dose methotrexate. AJNR Am J Neuroradiol 2005;26:1263-69

5. Reddick WE, Glass JO, Helton KJ, et al. A quantitative MRI assessment of leukoencephalopathy in children treated for acute lymphoblastic leukemia without irradiation. AJNR Am J Neuroradiol 2005;26:2371-77

6. Reddick WE, Laningham FH, Glass JO, et al. Quantitative morphologic evaluation of magnetic resonance imaging during and after treatment of childhood leukemia. Neuroradiology 2007;49:889-904

7. Pui C-H, Relling MV, Sandlund JT, et al. Rationale and design of total therapy study XV for newly diagnosed childhood acute lymphoblastic leukemia. Ann Hematol 2004;83:124-26

8. Rueckert D, Frangi AF, Schnabel JA. Automatic construction of 3-D statistical deformation models of the brain using nonrigid registration. IEEE Trans Med Imaging 2003;22:1014-25

9. Zhang Y, Zuo P, Mulhern RK, et al. Brain structural abnormalities in survivors of pediatric posterior fossa brain tumors: a voxel-based morphometry study using free-form deformation. Neuroimage 2008;42:218-29

10. Lee JE, Chung MK, Lazar M, et al. A study of diffusion tensor imaging by tissue-specific, smoothing-compensated voxel-based analysis. Neuroimage 2009; $44: 870-83$

11. Rutgers DR, Toulgoat F, Cazejust J, et al. White matter abnormalities in mild traumatic brain injury: a diffusion tensor imaging study. AJNR Am J Neuroradiol 2008;29:514-19

12. Xie S, Xiao JX, Gong GL, et al. Voxel-based detection of white matter abnormalities in mild Alzheimer disease. Neurology 2006;66:1845-49

13. Genovese CR, Lazar NA, Nichols T. Thresholding of statistical maps in functional neuroimaging using the false discovery rate. Neuroimage 2002; 15:870-78

14. Chu WC, Chik KW, Chan YL, et al. White matter and cerebral metabolite changes in children undergoing treatment for acute lymphoblastic leukemia: longitudinal study with MR imaging and ${ }^{1} \mathrm{H}$ MR spectroscopy. Radiology 2003;229:659-69

15. Harila-Saari A, Paakko E, Vainionpaa L, et al. A longitudinal magnetic resonance imaging study of the brain in survivors of childhood acute lymphoblastic leukemia. Cancer 1998;83:2608-17

16. Hertzberg H, Huk WJ, Ueberall MA, et al. CNS late effects after ALL therapy in childhood. Part 1: neuroradiological findings in long-term survivors of childhood ALL - an evaluation of the interferences between morphology and neuropsychological performance. Med Pediatr Oncol 1997;28:387-400

17. Paakko E, Harila-Saari A, Vanionpaa L, et al. White matter changes on MRI during treatment in children with acute lymphoblastic leukemia: correlation with neuropsychological findings. Med Pediatr Oncol 2000;35:456-61

18. Lesnik PG, Ciesielski KT, Hart BL, et al. Evidence for cerebellar-frontal subsystem changes in children treated with intrathecal chemotherapy for leukemia. Arch Neurol 1998;55:1561-68

19. Carey ME, Haut MW, Reminger SL, et al. Reduced frontal white matter volume in long-term childhood leukemia survivors: a voxel-based morphometry study. AJNR Am J Neuroradiol 2008;29:792-97

20. Mabbott DJ, Noseworthy M, Bouffet E, et al. White matter growth as a mechanism of cognitive development in children. Neuroimage 2006;33:936-46

21. Campell LK, Scaduto M, Sharp W, et al. A meta-analysis of the neurocognitve sequelae of treatment for childhood acute lymphocytic leukemia. Pediatr Blood Cancer 2007;49:65-73

22. Moleski M. Neuropsychological, neuroanatomical, and neurophysiological consequences of CNS chemotherapy for acute lymphoblastic leukemia. Arch Clin Neuropsychol 2000;15:603-30

23. Mulhern RK, Wasserman AL, Fairclough D, et al. Memory function in diseasefree survivors of childhood acute lymphocytic leukemia given CNS prophylaxis with or without $\mathbf{1 , 8 0 0}$ cGy cranial irradiation. J Clin Oncol 1988;6:315-20

24. Nathan PC, Patel SK, Dilley K, et al. Guidelines for identification of, advocacy for, and intervention in neurocognitive problems in survivors of childhood cancer: a report from the Children's Oncology Group. Arch Pediatr Adolesc Med 2007;161:798-806

25. Hockenberry MJ, Krull KR, Moore K, et al. Longitudinal evaluation of fine motor skills in children with leukemia. J Pediatr Hematol Oncol 2007; 29:535-39

26. Brown RT, Madan-Swain A, Walco GA, et al. Cognitive and academic late effects among children previously treated for acute lymphocytic leukemia receiving chemotherapy as CNS prophylaxis. J Pediatr Psychol 1998;23: $333-40$ 
27. Montour-Proulx I, Kuehn SM, Keene DL, et al. Cognitive changes in children treated for acute lymphoblastic leukemia with chemotherapy only according to the Pediatric Oncology Group 9605 protocol. J Child Neurol 2005;20:129-33

28. Espy KA, Moore IM, Kaufmann PM, et al. Chemotherapeutic CNS prophylaxis and neuropsychologic change in children with acute lymphoblastic leukemia: a prospective study. J Pediatr Psychol 2001;26:1-9

29. Kaemingk KL, Carey ME, Moore IM, et al. Math weaknesses in survivors of acute lymphoblastic leukemia compared to healthy children. Child Neuropsychology 2004;10:14-23

30. Carey ME, Hockenberry MJ, Moore IM, et al. Brief report: Effect of intravenous methotrexate dose and infusion rate on neuropsychological function one year after diagnosis of acute lymphoblastic leukemia. J Pediatr Psychol 2007;32:189-93

31. Plotkin SR, Wen PY. Neurologic complications of cancer therapy. Neurol Clin NAm 2003;21:279-318

32. Basser PJ, Jones DK. Diffusion-tensor MRI: theory, experimental design and data analysis-a technical review. NMR Biomed 2002;15:456-67

33. Leung LH, Ooi G-C, Kwong DL, et al. White-matter diffusion anisotropy after chemo-irradiation: a statistical parametric mapping study and histogram analysis. Neuroimage 2004;21:261-68

34. Khong P-L, Leung LH, Fung AS, et al. White matter anisotropy in post-treatment childhood cancer survivors: preliminary evidence of association with neurocognitive function. J Clin Oncol 2006;24:884-90 